\title{
NEAR-OPTIMAL RE-ENTRY TRAJECTORIES FOR REUSABLE LAUNCH VEHICLES
}

\author{
H.-C. Chou* and M. D. Ardema ${ }^{\dagger}$ \\ Santa Clara University \\ Santa Clara, California 95053 \\ and
}

J. V. Bowles ${ }^{\ddagger}$

NASA Ames Research Center
Moffet Field, California 94035

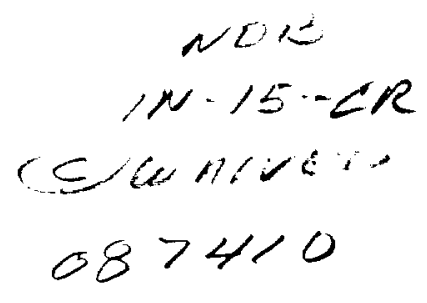

$N Q$

$g_{s} \quad=$ gravitational acceleration on the earth

$\underline{\text { Abstract }}$

A near-optimal guidance law for the descent trajectory for earth orbit re-entry of a fully reusable single-stage-to-orbit pure rocket launch vehicle is derived. A methodology is developed to investigate using both bank angle and altitude as control variables and selecting parameters that maximize various performance functions. The method is based on the energy-state model of the aircraft equations of motion. The major task of this paper is to obtain optimal reentry trajectories under a variety of performance goals: minimum time, minimum surface temperature, minimum heating, and maximum heading change; four classes of trajectories were investigated: no banking, optimal left turn banking, optimal right turn banking, and optimal bank chattering. The cost function is in general a weighted sum of all performance goals. In particular, the trade-off between minimizing heat load into the vehicle and maximizing cross range distance is investigated. The results show that the optimization methodology can be used to derive a wide variety of near-optimal trajectories.

\section{Nomenclature}

$$
\begin{array}{ll}
D & =\text { drag, } \mathrm{lb} \\
E & =\text { total mechanical energy per unit weight, } \mathrm{ft} \\
e & =\text { emissivity } \\
g & =\text { gravitational acceleration at altitude, } \mathrm{ft} / \mathrm{sec}^{2}
\end{array}
$$

$h \quad=$ altitude, $\mathrm{ft}$

$K=$ weighting parameter, $\mathrm{lb} / \mathrm{ft}^{3}$

$L \quad=$ lift, $\mathrm{lb}$

$M \quad=$ Mach number

$m \quad=$ aircraft mass, slugs

$R \quad=$ radius of the earth, $\mathrm{ft}$

$T_{\text {sur }} \quad=$ surface temperature, ${ }^{\circ} \mathrm{R}$

$t \quad=$ time, sec

$V \quad=$ speed, fps

$W \quad=$ aircraft earth surface weight (mass), $\mathbf{l b}$

$X \quad=$ longitudinal range over earth surface, $\mathrm{ft}$

$Y \quad=$ latitudinal range over earth surface, $\mathrm{ft}$

$\alpha \quad=$ angle of attack, rad

$\chi \quad=$ heading angle, $\mathrm{rad}$

$\phi \quad=$ bank angle, $\mathrm{rad}$

$\gamma \quad=$ flight-path angle, $\mathrm{rad}$

$\sigma=$ Boltzman constant, $\mathrm{Rho} / \mathrm{ft}^{2}-\mathrm{sec}-{ }^{\circ} \mathrm{R}^{4}$

$\omega=$ earth rotating angular velocity, $\mathrm{rad} / \mathrm{sec}$

\section{Introduction}

A national program is underway to identify the best launch vehicle and transportation architectures to make major reductions in cost of space transportation, while at the same time increasing safety for flight crews. Attention has focused on a single-stage-to-orbit, rocket powered, fully reusable launch vehicle (RVL). ${ }^{1.2}$ Missions being studied include satellite servicing and

surface, $\mathrm{ft} / \mathrm{sec}^{2}$

$q \quad=$ dynamic pressure, $\mathrm{lb} / \mathrm{ft}^{2}$

\footnotetext{
- Graduate Research Assistant, Department of Mechanical Engineering. Knowledge Engineer, Applied Materials, Inc. Student Member AIAA.

${ }^{t}$ Professor and Chairman, Department of Mechanical Engineering. Associate Fellow AIAA.

₹ Aerospace Engineer, System Analysis Branch. Member AIAA.
}

Copyright $(\mathcal{C} 1997$ by the American Institute of Aeronautics and Astronautics, INC. All right reserved. 
deployment and space station logistics delivery/return with flight crews rotation. This paper studies a optimal re-entry guidance law for such a vehicle.

One of the key objectives of re-entry trajectory optimization is to deliver the vehicle to the desired ground destination while limiting heat input and acceleration. The interest in turning trajectories arises from abort requirements, particularly the need to return to the launch site after one orbit. The hot environment to which the vehicle is exposed is one of the challenging problems for hypersonic flight control. Since high angles-of-attack required for turning increases the vehicle's surface temperatures, this abort trajectory is the case that typically sizes the thermal protection system. What is desirable is a flight path that results in sufficient cross-range while minimizing heating subject to temperature limits on the vehicle surface. A major goal of this paper is to determine trajectories that minimize heat input or turning capability, or a weighted combination of the two.

There has been significant recent research in trajectory optimization of hypersonic vehicles (Refs 36). In [3], Ronneke and Markl employ a linear feedback control law for trajectory control whose gain is scheduled with respect to the energy. Bradt et al. [4] propose autonomous guidance using a nonlinear programming algorithm with a selftargeting capability. Skakecki and Martin [5] use the optimization algorithm FAST (Flight Algorithm to Solve Trajectories) that combines nonlinear programming with a shooting method for two-point boundary-value problems to repeatedly calculate a set of command profiles that satisfy the mission constraints. Corban et al. [6] use energy-state method similar to those of the present paper. Important results and significant progress have been achieved. There have also been papers on heat loads and heat inputs during re-entry (Refs. 7-9).

Application of optimal control theory in the form of the maximum principle to aircraft trajectory optimization problems generally results in a two-pointboundary-value problem (2PBVP). The order of this problem is double the number of state variables and the equations are always "half unstable." Many schemes have been developed to numerically solve this difficult class of problem, but all are unsuitable in a vehicle synthesis study. Although there are well-developed numerical methods for trajectory optimization of pointmass vehicle models, these methods are too expensive computationally and not robust enough to use at conceptual design stage, in which many hundreds of vehicle designs must be evaluated and compared on a consistent basis.

What is needed in a vehicle synthesis study is a method that optimizes the trajectory in one pass, that is as an integral part of the trajectory integration. The method must also be robust and it should be easy to use and to interpret physically. The key to achieving this is to use judicious approximations that reduce the functional optimization problem to a function one.

In this paper, the Energy State Approximation (ESA) is used to obtain optimal trajectories. This wellknown technique substitutes the total mechanical energy for the speed as a state variable, and then neglects the altitude and flight path dynamics relative to the energy dynamics. Formally, this may be viewed as the use of Singular Perturbation Theory (SPT) to timescale decouple the equations of motion. When flight path optimization is done with the ESA model, simple rules for the optimal trajectory are obtained. This dynamic model has been used successfully many times to obtain effective guidance laws for a wide variety of aircraft and missions.

In a series of papers ${ }^{8,10-12}$ we have used energy-state methods to develop algorithms for ascent trajectory optimization and optimal operation of propulsion systems of advanced launch vehicles. The present paper extends these methods to enable computation of near-optimal re-entry trajectories for minimum time, minimum heat load, minimum surface temperature, maximum heading angle rate, or a combination of these.

In the numerical results, vehicle performance is computed using the NASA Ames hypersonic aircraft vehicle optimization code (HAVOC) ${ }^{13}$. HAVOC integrates geometry, aerodynamics, aerothermodynamics, propulsion, structures, weights, and other computations to produce point designs for a wide variety of launch vehicles. It is capable of iteratively sizing launch vehicles for specified missions. Although the trajectory guidance law is based on the energy-state model, the trajectory integration in HAVOC uses a point mass model, including the effects of earth rotation, earth curvature, and variable gravity.

\section{Methods}

The three-dimensional aircraft point-mass equations of motion over a spherical, rotating earth with no winds aloft, zero thrust, and terms in square of the earth rotation ignored are 


$$
\begin{aligned}
& \dot{X}=\frac{V R \cos \gamma \cos \chi}{(h+R) \cos \frac{Y}{R}} \\
& \dot{Y}=\frac{V R \cos \gamma \sin \chi}{(h+R)} \\
& \dot{h}=V \sin \gamma \\
& \dot{V}=\frac{-D}{m}-g \sin \gamma \\
& \dot{\gamma}=\left(\frac{L}{m V}\right) \cos \phi-\frac{g}{V} \cos \gamma+\frac{V}{(h+R)} \cos \gamma+2 \omega \cos \chi \cos \frac{Y}{R} \\
& \dot{\chi}=\left(\frac{L}{m V \cos \gamma}\right) \sin \phi-\frac{V}{(h+R)} \cos \gamma \cos \chi \tan \frac{Y}{R}+2 c\left(\tan \gamma \sin \chi \cos \frac{Y}{R}-\sin \frac{Y}{R}\right)
\end{aligned}
$$

For re-entry with thrust equal zero, the mass is constant. Define the aircraft energy per unit weight by

$$
E=\frac{h R}{R+h}+\frac{1}{2 g_{S}} V^{2}
$$

Differentiate and use the state equations in (1)

$$
\dot{E}=-\frac{V D}{m g_{S}}=P
$$

Where $P$ is the specific excess power.

Now replace $V$ by $E$ as state variable and use the observation that $h$ and $\gamma$ are capable of rapid change relative to $E$. The time rate of change of the flight path angle, $\dot{\gamma}$, and the altitude, $\dot{h}$, are then neglected. This gives $\gamma=0$ and the state equations become:

$$
\begin{aligned}
& \dot{X}=\frac{V R \cos \chi}{(h+R) \cos \frac{Y}{R}} \\
& \dot{Y}=\frac{V R \sin \chi}{(h+R)} \\
& \dot{E}=-\frac{V D}{m g}=P \\
& 0=\frac{L}{m V} \cos \phi-\frac{g}{V}+\frac{V}{(h+R)}+2 \omega \cos \chi \cos \frac{Y}{R} \\
& \dot{\chi}=\frac{L}{m V} \sin \phi-\frac{V}{(h+R)} \cos \chi \tan \frac{Y}{R}-2 \omega \sin \frac{Y}{R}
\end{aligned}
$$

We will use this approximation, the ESA, throughout.

As mentioned earlier, this approximation has a long history of successful application in a wide variety of flight trajectory problems. The main drawback is that the variables $h$ and $\gamma$ may now jump instantaneously at points along the trajectory, as well as at the boundaries. These jumps could be accounted for by boundary layer analysis, but this is not done in this paper.

\section{$\underline{\text { Minimum Time }}$}

To minimize the time to climb between two different altitudes and velocities, with no boundary conditions specified on $X, Y$, or $\chi$, using the energystate approximation, it is clear that we must maximize $\dot{E}$ with respect to $V$ for a given $E$. The quantity to be minimized for a given energy gain is

$$
J^{\prime}=\int_{i \infty}^{\prime \prime} d t=\int_{E=}^{E_{1}} \frac{1}{P} d E
$$

where Eq.(3) was used. It is assumed that $P$ is negative, $\mathrm{E}$ is monotonic, and bank angle is zero.

For convenience, we choose to invert the integrand in Eq.(5) and maximize; the quantity to be maximized is

$$
J=\int_{E_{0}}^{E_{f}} P d E
$$

and thus the solution reduces to

$$
\left.\max _{h}(P)\right|_{E=\text { comst }}
$$

subject to the fourth of Eqs.(4) and $\phi=0$ (no banking). This is the well-known energy climb path.

Banking trajectories generally have higher lift and drag and thus may be expected to give shorter descent times. The optimization criterion in this case is

$$
\left.\max _{h . \phi}(P)\right|_{E=\text { consts }}
$$

A special case of banking is bank-chattering (rapid switches in bank angle that leave $\sin \phi=0$ ). The shuttle, for example, uses this technique. Now the criterion is

$$
\left.\underset{\substack{\max \\ \sin \&=0}}{\min }(P)\right|_{E=\text { romsi }}
$$

\section{Minimum Heat load}


The quantity to be minimized for a given energy gain in minimizing heat load is

$$
J^{\prime}=\int_{i 0}^{n} \dot{Q} d t=\int_{E_{0}}^{E} \frac{\dot{Q}}{P} d E
$$

where Eq.(3) was used and $Q$ is the total heat input at vehicle surface which may be defined as ${ }^{14}$

$$
\dot{Q}=e\left(T_{\text {suf }}\right) \sigma\left(T_{\text {surf }}{ }^{4}-T_{\text {ref }}{ }^{4}\right)
$$

Since $T_{\text {surf }}^{4} \gg T_{\text {ref }}^{4}$, for convenience, we simplify Eq.(11) by setting

$$
\dot{Q}=e \sigma T_{\text {suf }}{ }^{4}
$$

and choose to invert the integrand in Eq.(10) and maximize; from Eq.(10) and (12), the quantity to be maximized is

$$
J^{\prime}=\int_{E_{0}}^{\varepsilon_{f}} \frac{P}{T_{\text {surf }}{ }^{4}} d E
$$

and thus the solution reduces to

$$
\max \left(\frac{P}{T_{\text {soof }}^{4}}\right) \mid E=\text { coms }
$$

with $\phi=0$ for no-banking trajectories. The optimization criterion in banking trajectories is therefore

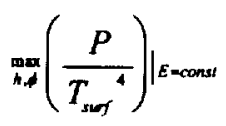

and in the case of bank-chattering the optimization problem is

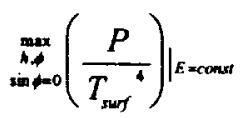

as before, the fourth of Eqs.(4) is enforced.

\section{Maximum Turn}

In order to return to the launch site, for example in abort trajectories after one orbit, it is necessary that the launch vehicle be capable of executing turning trajectories with significant crossrange. Inspection of Eqs.(4), however, shows that if cross-range is to be maximized then both $E$ and $\chi$ must be retained as state variables and the ESA approximation is not valid. Instead, to investigate turning capability we consider maximum heading angle change trajectories.

For maximum heading angle change

trajectories, there is still coupling between the $\dot{E}$ and $\dot{\chi}$ state equations. The coupling occurs in the earth curvature (centripetal) terms and in the earth rotation (Coriolis) terms. Although the latter are relatively small effects, the centripetal terms are relatively large at the start of re-entry from orbit. Both of these types of terms must be ignored to obtain the ESA approximation. The quantity to be maximized is then

$$
J^{\prime}=\int_{\chi^{0}}^{x^{\prime}} d \chi=\int_{10}^{11} \dot{\chi} d t=\int_{E_{01}}^{E_{0}} \frac{\dot{\chi}}{P} d E
$$

Where $\dot{\chi}$ is given by the last of Eqs.(4). In this case the optimization problem is therefore

$$
\max _{h, \phi}\left(\frac{P}{-\dot{\chi}}\right)_{E=\text { const }}
$$

If the heading change reaches $90^{\circ}$ relative to the deorbit value, a maximum range glide is initiated.

Maximum turning trajectories may result in excessive heating. For this reason, trajectories maximizing a weighted sum of heading change and the negative of heat input were also considered. In this case, the optimization problem is

$$
\left.\max _{h, \phi}\left(\frac{P}{K_{1} T_{\text {suf }}{ }^{4}-K_{2} \dot{\chi}}\right)\right|_{E=\text { const }}
$$

where $K_{1}$ and $K_{2}$ are mission dependent weighting parameters to be determined empirically.

\section{Numerical Results}

All numerical examples are based on an SSTO rocket with a delta winged-body configuration ${ }^{2}$. The 
vehicle takes off vertically and lands horizontally. The minimum and maximum dynamic pressure limits were 20 and $900 \mathrm{psf}$, respectively. The temperature constraints at a point on the windward side of the vehicle $1 / 3$ of the way back from the nose are $1200^{\circ} \mathrm{F}$ for the upper surface and $1900^{\circ} \mathrm{F}$ for the lower surface. No stagnation heating constraints were imposed. The angle of attack was limited to $\mathbf{4 5} \mathrm{deg}$ and the maximum load factor was set to 2.5. The first results to be presented are minimum time re-entry trajectories.

\section{$\underline{\text { Minimum Time }}$}

Figures 1-8 illustrate the results from minimizing time. Four classes of trajectories were investigated: no banking, optimal left turn banking, optimal right turn banking, and optimal bank chattering (see Eqs.(6-8)).

Figure 1 shows that the banking trajectories have much lower dynamic pressure than does the nonbanking one, and the latter one generally follows the maximum dynamic pressure (q) boundary. Figure 2 shows the cost function $(P)$ as a function of altitude and Mach number in the flight envelope for the no banking case (zero cost indicates constrains are violated). It is seen that there is another local optimal at very low q. Figure 3 shows vehicle lower surface temperatures at a point $1 / 3$ back of the vehicle nose on the windward side, and these temperature contours are shown in the flight envelop in Figure 4. Many of these temperatures are higher than allowable for the preferred thermal protection system, and thus temperature limits must be imposed on the trajectory. These figures show that there are two local minimums in the hypersonic region, one just above the $q_{\max }$ boundary and one just below the $\mathrm{q}_{\min }$ boundary.

Figure 5 compares minimum-time no-banking, banking and bank-chattering trajectories and shows that the ground path is considerably shortened if banking or chattering is allowed. The coordinates used are curvilinear down-range and cross-range. Table 1 shows that banking or bank-chattering shortens the reentry time by about 200 seconds.

Figure 6 shows that the angle-of-attack of the banking trajectories is much higher than for the nonbanking ones, about $45^{\circ}$ as compared with $5^{\circ}$. The bank angle of the banking trajectories is in the range of $60^{\circ}-80^{\circ}$ (Figure 7 ).

Figure 8 shows the history of the lower surface temperature at the point on the windward side of the vehicle $1 / 3$ of the way back from the nose. For all the minimum time trajectories, this temperature is mostly at $1900^{\circ} \mathrm{F}$, the upper imposed limit, for a significant portion of the trajectory.

\section{Minimum Temperature}

The minimum temperature ( $1 / 3$ back on windward side) trajectories are shown in Figures 9-13. Comparison with the minimum time paths of Figures 1 8 show significant differences. The minimum temperature trajectories are significantly longer in both range and time ( $5240 \mathrm{n} . \mathrm{mi}$. down range as compared with $1730 \mathrm{n} . \mathrm{mi}$. at most, $2100 \mathrm{sec}$. as compared with $660 \mathrm{sec}$.); they also have relatively low dynamic pressure. When allowed to bank or bank-chatter, there is a little banking at the end, where temperature is not a factor; at high speed, there is no banking, which is to be expected, since banking increases angle-of-attack. The maximum temperature is greatly reduced relative to the minimum time paths $\left(1400^{\circ} \mathrm{F}\right.$ as compared with $\left.1900^{\circ} \mathrm{F}\right)$ and the time at high temperature is significantly reduced. A significant portion of the minimum temperature trajectories are near the maximum angle of attack of $45^{\circ}$.

\section{Minimum Heating}

One of the key objectives of a re-entry trajectory is minimizing heat input into the thermal protection system. In this paper the heat input has been approximated as the integral of vehicle surface temperature to the fourth power over time (proportional to the convective heating rate). Thus the minimum heating trajectories would be expected to be in some sense between the minimum time and the minimum temperature ones, tending more towards minimum temperature. Figures 14-18 show, in fact, that the minimum heating trajectories are similar to the minimum temperature ones, but with less range, shorter time, and more time at maximum angle of attack; small amounts of banking and bank-chattering are optimal. Comparison of Figures 9 and 14 show that the minimum heating trajectories are also very nearly minimum temperature trajectories.

\section{Maximum Heading Change}

Maximum heading angle change trajectories are shown on Figures 19-23. The interest in turning trajectories arises from abort requirements, particularly the need to return to the launch site after one orbit. Since turning increases vehicles surface temperatures, this abort trajectory is the case that typically sizes the 
thermal protection system. What is desirable is a flight path that results in sufficient cross-range while minimizing heating subject to temperature limits on the vehicle surface. As mentioned earlier, maximum crossrange problems do not reduce to energy-state approximation, and therefore maximum heading change trajectories are determined instead as an approximation. (When heading change reaches $90^{\circ}$, it is held constant.) Although there is coupling between the $\dot{E}$ and $\dot{\chi}$ equations for maximum cross range, it is only through the centripetal and Coriolis terms, as mentioned earlier.

Figure 20 shows the ground paths for left and right maximum heading change trajectories. A representative zero bank trajectory is shown for comparison. Because of the earth curvature and the use of curvilinear coordinates, the zero bank trajectory has a curved ground path. Both left and right turns are relatively hard turns. They begins at high angle-ofattack, about $30^{\circ}$, and then switch to about $15^{\circ}$ (Figure 21 ); the bank angle is at $60^{\circ}-70^{\circ}$ for most of the flight (Figure 22). The right hand turn takes considerably more time than the left (Table 1). For both trajectories, the surface temperature is at the $1900^{\circ} \mathrm{F}$ limit for a considerable time (Figure 23). It is of interest to note that $15^{\circ}$ is the angle-of-attack for maximum lift-to-drag ratio. Thus the latter portion of these trajectories are flown at maximum $L / D$. This is in agreement with the classical result that maximum range in gliding flight is attained at maximum $\mathrm{L} / \mathrm{D}$.

In Figures 24 and 25, a comparison of the heat load as a function of cross range distance is made for optimal right turn banking and optimal left turn banking. As shown in Figure 24 (right turn banking), cross range distance of up to $400 \mathrm{n} . \mathrm{mi}$. can be achieved without significantly increasing heat load. For the case of left turn banking, Figure 25, cross range of up to $1200 \mathrm{n} . \mathrm{mi}$. is achievable without significant increase in heat input.

\section{Concluding Remarks}

A cost function based on energy-state approximation was used to derive a near-optimal guidance law for re-entry trajectories of a single-stageto-orbit all rocket launch vehicle. The guidance law was employed to obtain optimal re-entry trajectories for minimum time, minimum heat load, maximum heading angle change, and a combination of these.
A vehicle synthesis code using the guidance law was used to investigate SSTO re-entry mission performance. Numerical results show that minimum time trajectories are quite different than minimum temperature trajectories. The former have much shorter times, much higher dynamic pressures, much higher vehicle surface temperatures and use banking, as compared with the latter.

Minimum heating trajectories are intermediate between minimum time and minimum temperature, being more like the latter. Maximum heading rate change trajectories were also computed; they are mostly at maximum lift-to-drag ratio with large values of bank angle. Finally, trajectories minimizing a weighted sum of heat input and negative cross-range were considered. It was found that significant crossrange is possible without significantly increasing heat input.

In addition to being a useful tool in preliminary design studies, the guidance algorithm should be ideal for use in an on-board real-time control system because:(1) it is fully nonlinear and models all of the vehicle's significant nonlinearities, (2) it is algebraic and thus does not rely on potentially unstable numerical integrations, and (3) it depends directly on easily measured vehicle states and parameters.

\section{Acknowledgment}

This work was supported by NASA Ames Research Center Grant NCC 2-5069.

\section{References}

'Bekey, I., "SSTO Rockets; A Practical Possibility," Aerospace America, July 1994, pp.32-37.

${ }^{2}$ Freeman, D. C., Talay, T. A., Stanley, D. O., Lepsch, R. A., and Wilhite, A. W., "Design Options for Advanced Manned Launch Systems," Journal of Spacecraft and Rockets, Vol.32, No. 2, March-April 1995, pp.241-249.

${ }^{3}$ Roenneke, A. J., and Markl, A., "Reentry Control of a Drag vs. Energy Profile," Journal of Guidance, Control, and Dynamics, Vol. 17, No. 5, 1994, pp.916-920.

${ }^{4}$ Bradt, J., Langehough, M., Robert, R., "Autonomous Guidance and Control for a Low L/D Crew Return Vehicle," AIAA Guidance, Navigation, 
and Control Conference, New Orleans, LA, Aug. 1991, AIAA 91-2819

${ }^{5}$ Skalecki. L., Martin, M., "General Adaptive Guidance Using Nonlinear Programming ConstraintSolving Methods," Journal of Guidance, Control, and Dynamics, Vol. 16, No. 3, May-June 1993, pp.517-522.

${ }^{6}$ J. E. Corban, A. J. Calise, and G. A. Flandro, "Rapid Near-Optimal Aerospace Plane Trajectory Generation and Guidance," Journal of Guidance, Control, and Dynamics, Vol. 14, 1991, pp.1181-1190.

${ }^{7}$ G. Sachs and M. Dinkelmann, "Heat Input Reduction in Hypersonic Flight by Optimal Trajectory Control," AIAA Guidance Navigation and Control Conference, July 29-31, 1996, San Diego, CA.

${ }^{8}$ Ardema, M. D., Bowles, J. V., Terjesen, E. J., and Whittaker, T., "Near-Optimal Propulsion System Operation for Air-Breathing Launch Vehicles," AIAA Paper 94-3635, presented at the 1994 AIAA Guidance, Navigation, and Control Conf., Scottsdale, AZ, AUG. 1-3, 1994.

${ }^{9}$ C. Jansch and A. Markl, "Trajectory

Optimization and Guidance for a Hermes-Type Reentry Vehicle,"In AIAA-91-2659, 1991.

${ }^{10}$ Ardema, M. D., Bowles, J. V., and Whittaker, T., "Optimal Trajectories for Hypersonic Launch Vehicles," Dynamics and Control, Vol. 4, No. 4, 1994, pp.337-347.

${ }^{11}$ Ardema, M. D., Bowles, J. V., Terjesen, E. J., and Whittaker, T., "Approximate Altitude Transitions for High-Speed Aircraft," Journal of Guidance, Control, and Dynamics, Vol. 18, No. 3 , May-June 1995, pp.561-566.

${ }^{12}$ Ardema, M. D., Chou, H.-C., and Bowles, J. V., "Near-Optimal Operation of Dual-Fuel Launch Vehicles," Journal of Guidance, Control, and Dynamics, Vol. 19, No. 5, Sep.-Oct. 1996, pp.11801182.

${ }^{13}$ Bowles, J. V., “Ames Conceptual Studies Activities," Proceedings of the Second National Aerospace Plane Symposium, Applied Physics Lab., Laurel, MD, Nov. 1986.

${ }^{14}$ Windhorst, R., Ardema, M. D., Bowles, J. V., "Minimum Heating Re-entry Trajectories for
Advanced Hypersonic Launch Vehicles," AIAA Paper 97-3535, to be presented at the 1997 AIAA Guidance, Navigation and Control Conference. 


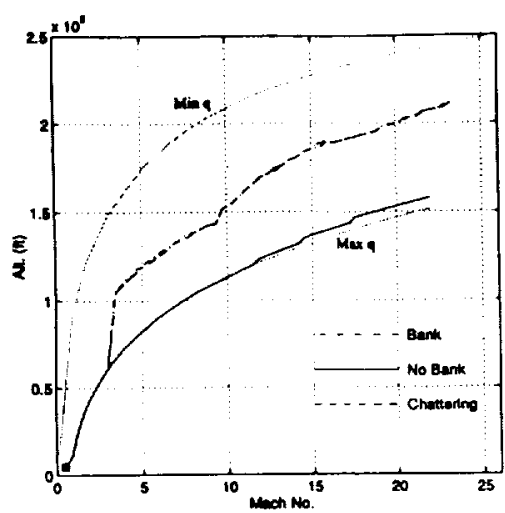

Figure 1 Minimum time re-entry trajectories of SSTO all-rocket launch vehicle.

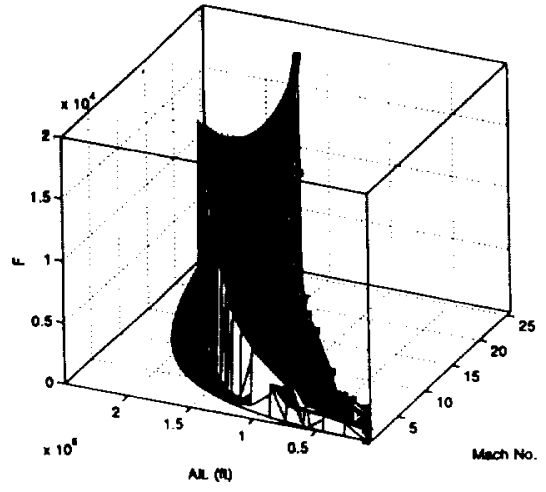

Figure 2 Cost function surface and optimized flight path of the min. time no banking case.

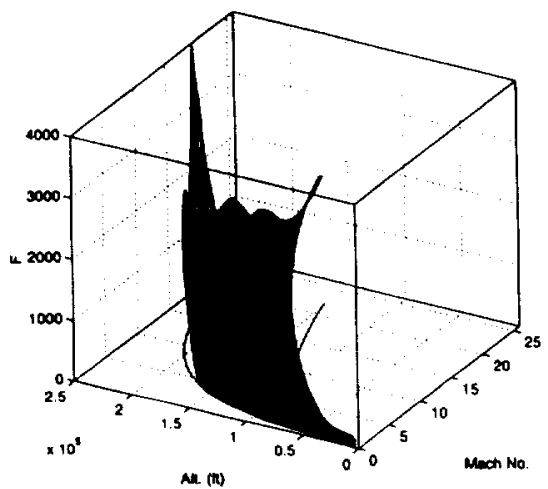

Figure 3 Temperature mesh ( $1 / 3$ back on windward side) of the min time no banking case.

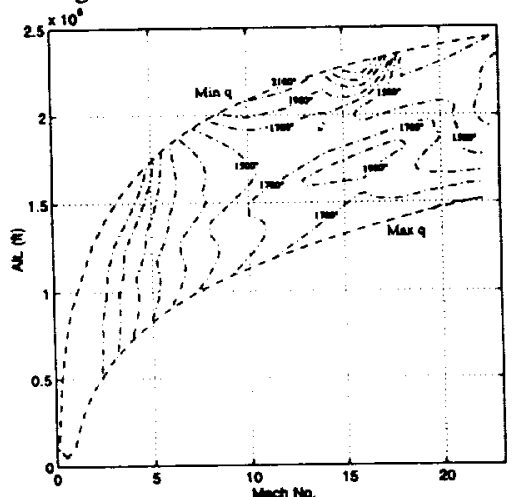

Figure 4 Temperature contours ( $1 / 3$ back on windward side) of the min. time no banking case.

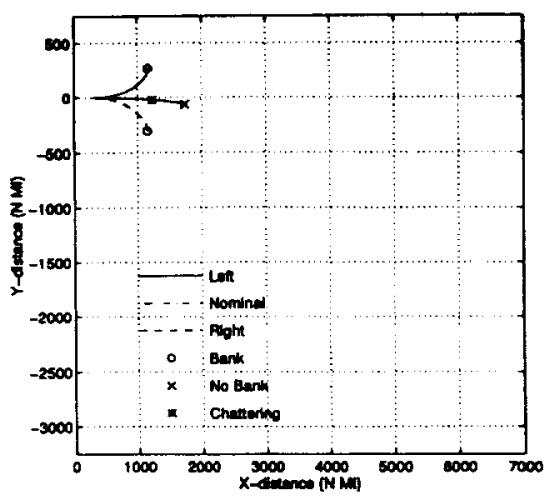

Figure 5 Ground cross distances for min. time descent.

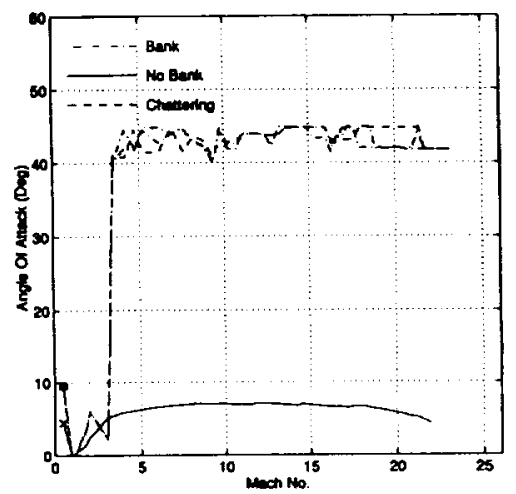

Figure 6 Time histories of angle of attack for min. time.

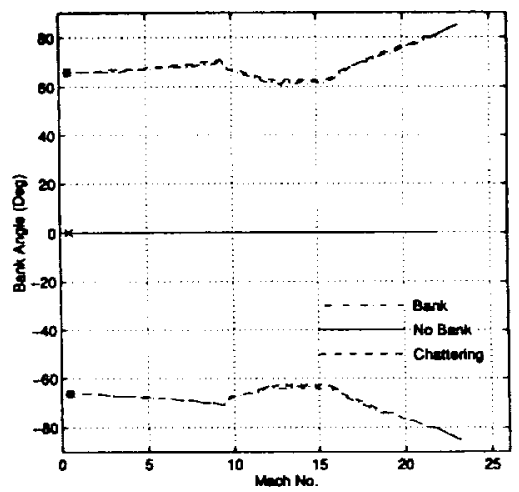

Figure 7 Time histories of bank angle for min. time.

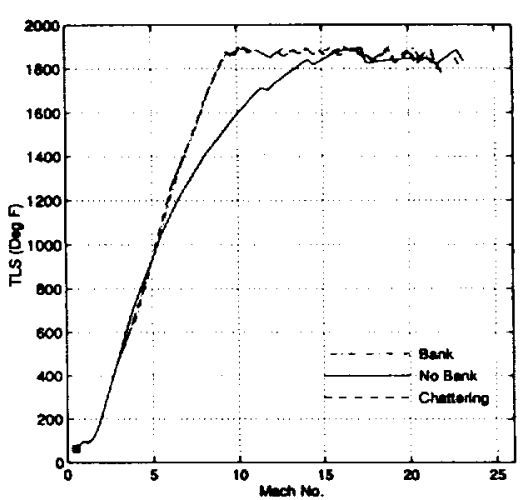

Figure 8 Histories of the lower surface temperature (1/3 back on windward side) for min. time. 


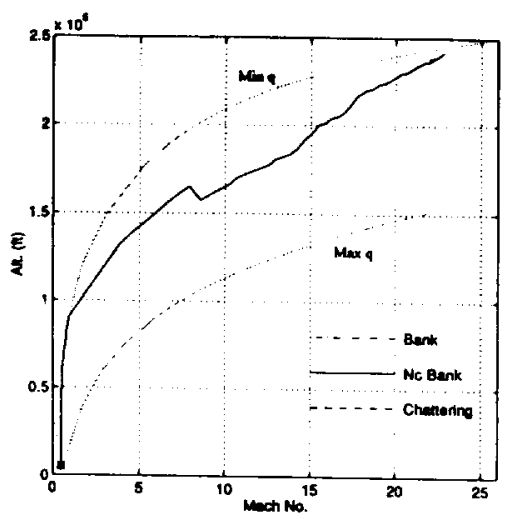

Figure 9 Re-entry trajectories of SSTO all-rocket launch vehicle for min. temperature ( $1 / 3$ back on windward side).

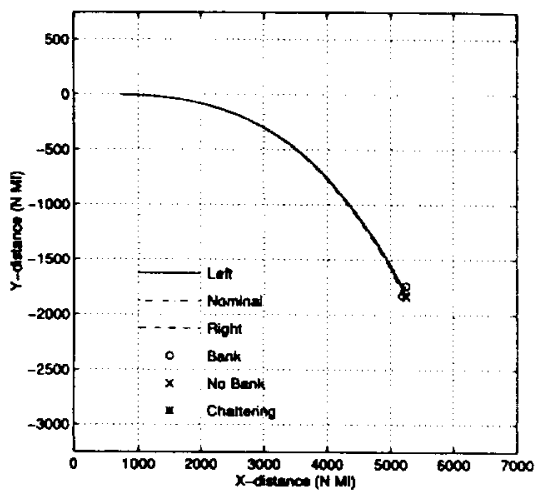

Figure 10 Ground cross distances for min. temperature.

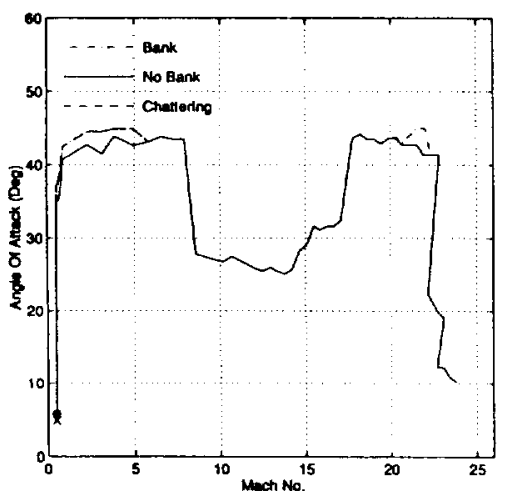

Figure 11 Time histories of angle of attack for min. temperature

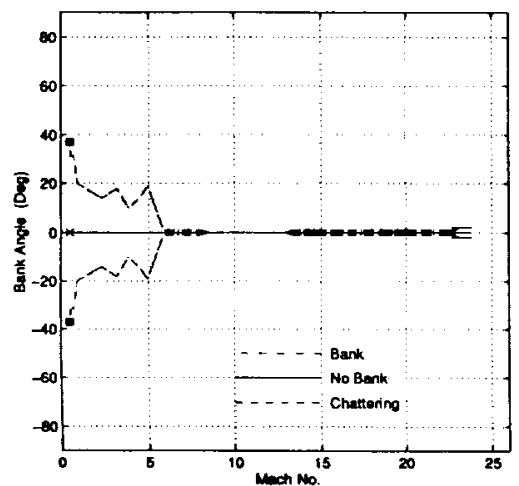

Figure 12 Time histories of bank angle for min. temperature.

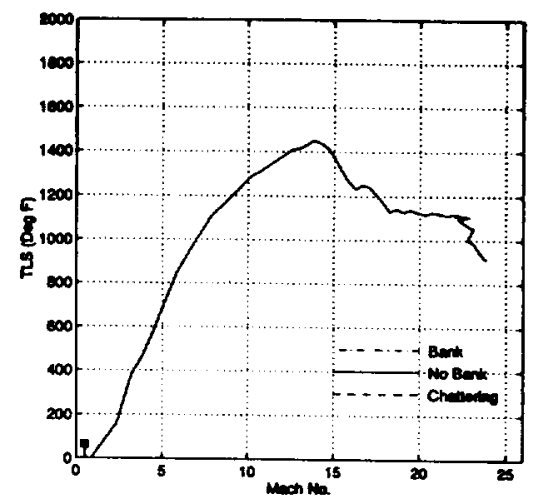

Figure 13 Histories of the lower surface temperature (1/3 back on windward side) for min. temperature.

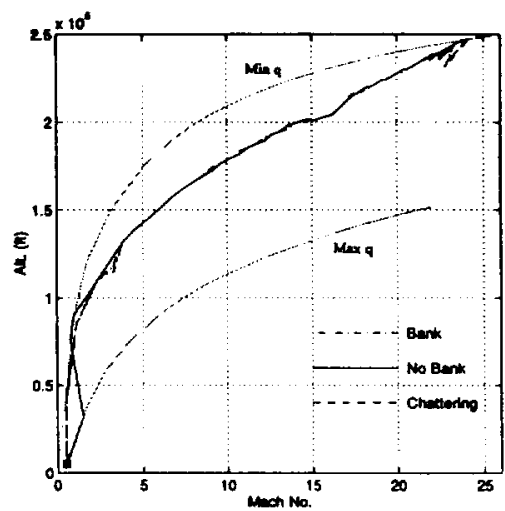

Figure 14 Re-entry trajectories of SSTO all-rocket launch vehicle for min. heat input.

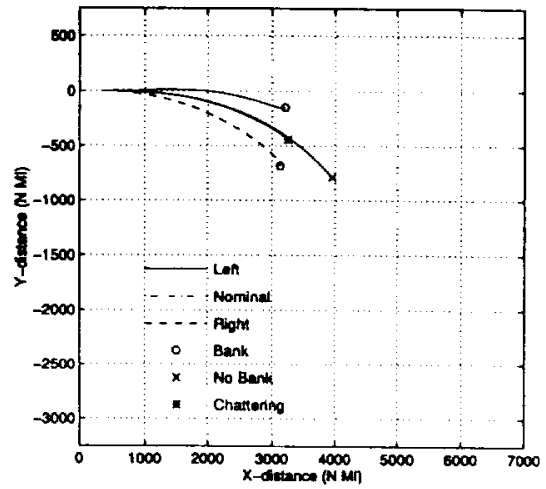

Figure 15 Ground cross distances for min. heat input.

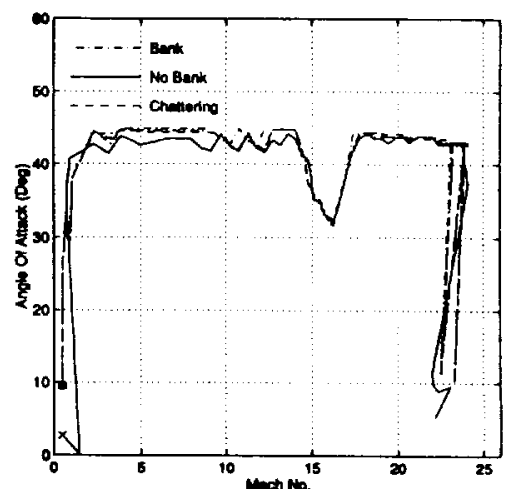

Figure 16 Time histories of angle of attack for min. heat input. 


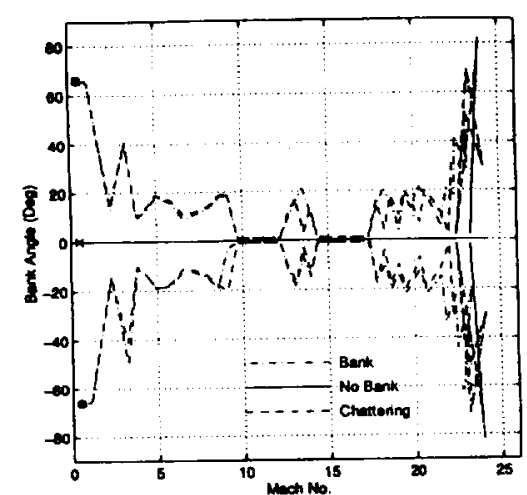

Figure 17 Time histories of bank angle for min. heat input.

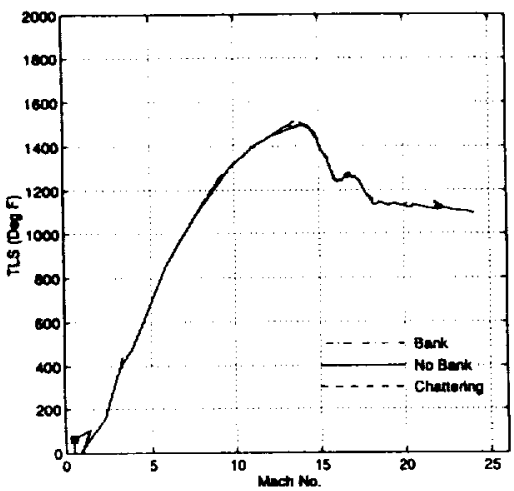

Figure 18 Histories of the lower surface temperature ( $1 / 3$ back on windward side) for min. heat input.

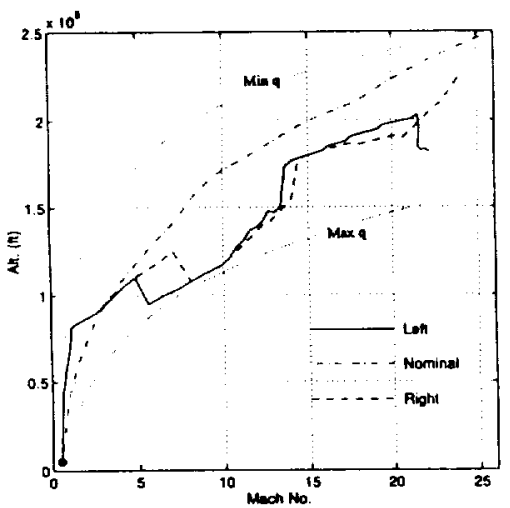

Figure 19 Re-entry trajectories of SSTO all-rocket launch vehicle for max. heading angle change.

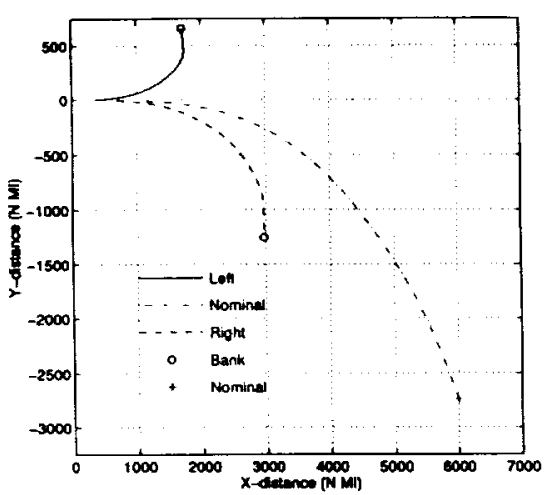

Figure 20 Ground cross distances for max. heading angle change.

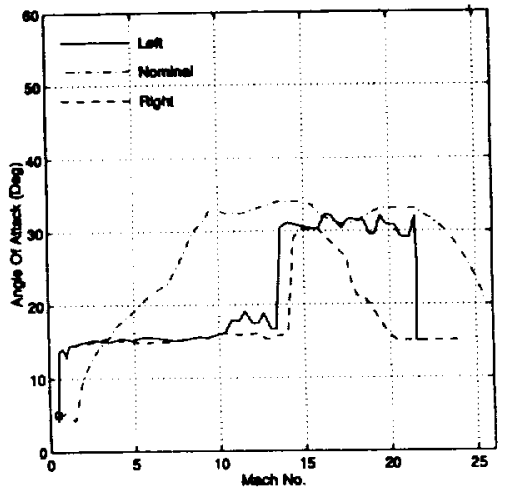

Figure 21 Time histories of angle of attack for max. heading angle change.

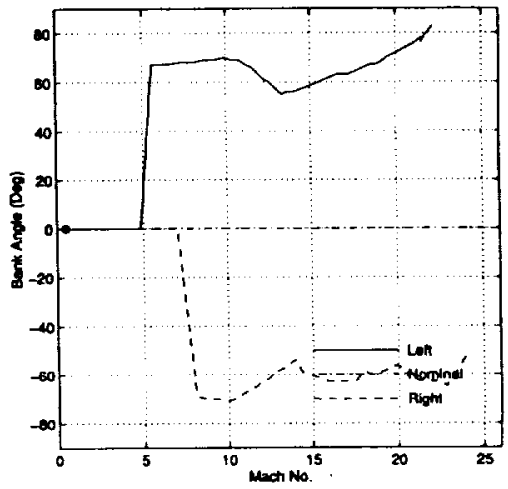

Figure 22 Time histories of bank angle for max. heading angle change.

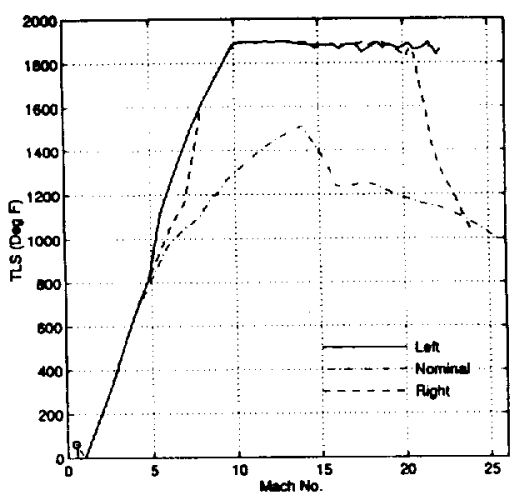

Figure 23 Histories of the lower surface temperature ( $1 / 3$ back on windward side) for max. heading angle change.

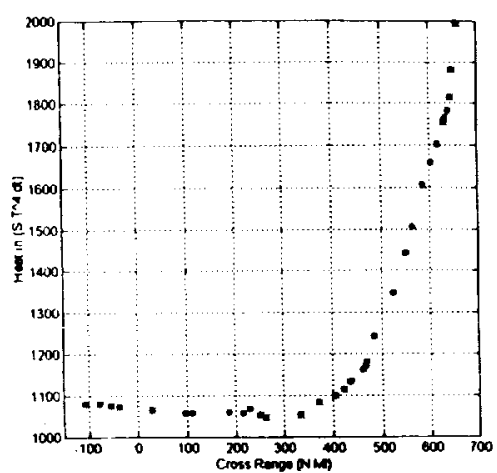

Figure 24 Variation of heat load and cross range with weighting parameter $\mathrm{K}_{2}$ for optimal right turn banking. 


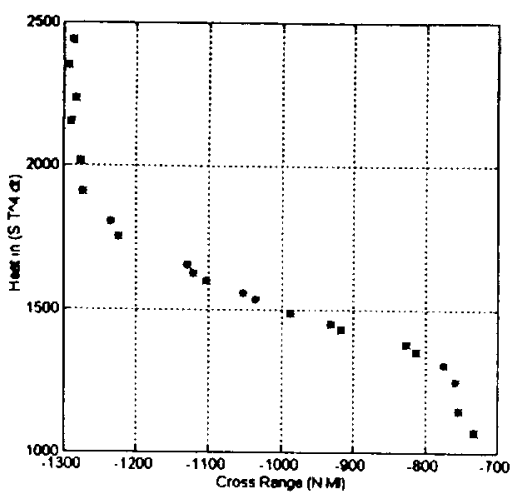

Figure 25 Variation of heat load and cross range with weighting parameter $\mathrm{K}_{2}$ for optimal left turn banking.

\begin{tabular}{|c|c|c|c|}
\hline Criteria & $S d t(S e c)$ & $S T^{\wedge} 4 \mathrm{dt}\left(\operatorname{Sec} \times \operatorname{Deg}^{\wedge} 4\right)$ & Cross Range (n Mille) \\
\hline Min Tlme - No Bank & 661.00 & $1.56 \mathrm{E}+16$ & 65.20 \\
\hline Min Time - Free Bank & 462.60 & $1.15 E+16$ & 268.20 \\
\hline Min Time - Chattering & 456.00 & $1.13 E+16$ & -22.40 \\
\hline Min. Temperature - No Bank & 2086.80 & $1.36 \mathrm{E}+16$ & -1837.60 \\
\hline Min. Ṫemperature -Free Bank & 2013.80 & $1.3 \overline{6 E+16}$ & -1738.50 \\
\hline Min. Temperature - Chattering & 201280 & $1.36 \mathrm{E}+16$ & .1820 .40 \\
\hline Min Heat Input - No Bank & 1478.20 & $1.16 E+16$ & -788.30 \\
\hline Min Heat Input - Froe Bank & 1327.20 & $1.08 \mathrm{E}+16$ & -145.90 \\
\hline Min Heat Input - Chattering & 1345.90 & $1.09 \mathrm{E}+16$ & -441.50 \\
\hline Max Heading Rate-Free Bank & 1255.20 & $2.01 E+16$ & 661.10 \\
\hline
\end{tabular}

Negative Banking (Rlght Turn)

\begin{tabular}{|c|c|c|c|}
\hline Criterla & Sdt (Sec) & $S T^{\wedge} 4 \mathrm{dt}\left(\operatorname{Sec} \times \operatorname{Deg}^{\wedge} 4\right)$ & Cross Range (n Milo) \\
\hline Min Time - No Bank & 661.00 & $1.56 \overline{\mathrm{E}}+15$ & -65.20 \\
\hline Min Time - Free Bank & 462.20 & $1.15 \mathrm{E}+16$ & .303 .70 \\
\hline Min Time-Chattering & 456.00 & $1.13 E+16$ & -22.40 \\
\hline Min. Temperature - No Bank & 2086.80 & $1.36 E+16$ & -1837.60 \\
\hline Min. Temperature - Free Bank & 2007.00 & $1.35 \mathrm{E}+16$ & -1824.20 \\
\hline Min. Temperature - Chattering & 2012.80 & $1.36 \mathrm{E}+16$ & -1820.40 \\
\hline Min Heat Input - No Bank & 1478.20 & $1.16 \mathrm{E}+16$ & .788 .30 \\
\hline Min Heat Inpul - Free Bank & 1325.90 & $1.08 E+16$ & -682.70 \\
\hline Min Heat Input - Chattering & 1345.90 & $1.09 E+16$ & -441.50 \\
\hline Max Heading Rate- Free Bank & 1689.60 & $2.49 \mathrm{E}+16$ & -1254.80 \\
\hline
\end{tabular}

Table 1 Endpoint status of the optimized cases 
